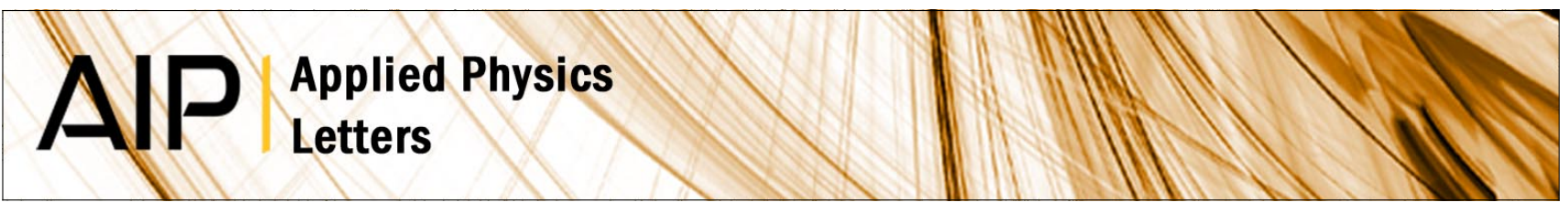

\title{
Reflectance anisotropy of Gd5Si2Ge2 and Tb5Si2.2Ge1.8
}

S. J. Lee, J. M. Park, J. E. Snyder, D. C. Jiles, D. L. Schlagel et al.

Citation: Appl. Phys. Lett. 84, 1865 (2004); doi: 10.1063/1.1687463

View online: http://dx.doi.org/10.1063/1.1687463

View Table of Contents: http://apl.aip.org/resource/1/APPLAB/v84/i11

Published by the American Institute of Physics.

\section{Related Articles}

The magnetic and magnetocaloric properties of NdFe12-xMox compounds

J. Appl. Phys. 111, 07A949 (2012)

Structural and magnetic study of SmTAl single crystals ( $\mathrm{T}=\mathrm{Pd}$ and $\mathrm{Ni})$

J. Appl. Phys. 111, 07E146 (2012)

Scatter in nonlinear ultrasonic measurements due to crystallographic orientation change induced anisotropy in harmonics generation

J. Appl. Phys. 111, 054905 (2012)

Structures and magnetic properties of Sm5Fe17 melt-spun ribbon

J. Appl. Phys. 111, 07E322 (2012)

Crystal structure of Tb5Ni2 In4 and Y5Ni2 In4, and magnetic properties of Dy5Ni2 In4

J. Appl. Phys. 111, 07E122 (2012)

\section{Additional information on Appl. Phys. Lett.}

Journal Homepage: http://apl.aip.org/

Journal Information: http://apl.aip.org/about/about_the_journal

Top downloads: http://apl.aip.org/features/most_downloaded

Information for Authors: http://apl.aip.org/authors

\section{ADVERTISEMENT}

\section{(e) ACCELERATE AMBER AND NAMD BY $5 X$. NVIDIA TRYIT ONA FREE, REMOTELYYHOSTED CLUSTER.}




\title{
Reflectance anisotropy of $\mathrm{Gd}_{5} \mathrm{Si}_{2} \mathrm{Ge}_{2}$ and $\mathrm{Tb}_{5} \mathrm{Si}_{2.2} \mathrm{Ge}_{1.8}$
}

\author{
S. J. Lee ${ }^{\text {a) }}$ \\ Ames Laboratory, Iowa State University, Ames, Iowa 50011 \\ J. M. Park \\ Department of Physics and Astronomy, Iowa State University, Ames, Iowa 50011 \\ J. E. Snyder and D. C. Jiles \\ Ames Laboratory and Materials Science and Engineering, Iowa State University, Ames, Iowa 50011 \\ D. L. Schlagel, T. A. Lograsso, and A. O. Pecharsky \\ Ames Laboratory, Iowa State University, Ames, Iowa 50011 \\ D. W. Lynch \\ Ames Laboratory and Department of Physics and Astronomy, Iowa State University, Ames, Iowa 50011
}

(Received 18 June 2003; accepted 26 January 2004)

Reflectance difference (RD) spectra for the $a-b$ plane of the single crystals of $\mathrm{Gd}_{5} \mathrm{Si}_{2} \mathrm{Ge}_{2}$ and $b-c$ planes of $\mathrm{Gd}_{5} \mathrm{Si}_{2} \mathrm{Ge}_{2}$ and $\mathrm{Tb}_{5} \mathrm{Si}_{2.2} \mathrm{Ge}_{1.8}$ were obtained in the photon energy range of $1.5-5.5 \mathrm{eV}$. Several peaks were observed for these crystals in the measured spectrum range. Similar features were observed in the RD spectra for the $b-c$ planes of $\mathrm{Gd}_{5} \mathrm{Si}_{2} \mathrm{Ge}_{2}$ and $\mathrm{Tb}_{5} \mathrm{Si}_{2.2} \mathrm{Ge}_{1.8}$, while different features were observed for the $a-b$ plane and $b-c$ plane of $\mathrm{Gd}_{5} \mathrm{Si}_{2} \mathrm{Ge}_{2}$. The RD spectra for the crystals arise not only from the surface anisotropy but also from the bulk anisotropy due to the monoclinic structure of the bulk crystal. (C) 2004 American Institute of Physics.

[DOI: $10.1063 / 1.1687463$ ]

Research on the electronic, magnetic, thermal, and mechanical properties of the $\mathrm{Gd}_{5}\left(\mathrm{Si}_{x} \mathrm{Ge}_{1-x}\right)_{4}$ intermetallic system, and in particular, the region around $\mathrm{Gd}_{5} \mathrm{Si}_{2} \mathrm{Ge}_{2}$, has recently increased due to their unique fundamental physical properties such as the existence of a magnetic-martensitic first-order phase transformation, the giant magnetocaloric effect, colossal magnetostriction $(\sim 10000 \mathrm{ppm})$, and giant magnetoresistance $(\sim 25 \%) .{ }^{1-5}$ Useful future applications can be envisioned from utilizing these impressive properties, such as room temperature magnetic refrigeration technologies using the giant magnetocaloric effect properties, noncontact force torque sensors and actuators using the colossal magnetostriction, and the magnetoelectrical devices using the giant magnetoresistance. However, much remains to be understood concerning their fundamental physical properties and relationship to structure.

Theoretical electronic structure calculations have been performed with linear muffin-tin orbital method based on the local density approximation (LDA) and LDA $+\mathrm{U}$ as attempts to understand the nature of unique properties of $\mathrm{Gd}_{5} \mathrm{Si}_{2} \mathrm{Ge}_{2} .{ }^{6-8}$ Validation of theoretical calculation can be achieved from comparison with experimental data. Reflectance difference spectroscopy (RDS) is a sensitive optical probe for investigating optical anisotropy properties of a material. ${ }^{9-11}$ RDS measures the optical reflectance difference, $\Delta R$, between the normal-incidence reflectance $R_{x}$ and $R_{y}$ for two orthogonal polarization directions in the surface plane. $R_{x}$ and $R_{y}$ from the bulk of a cubic crystal are the same due to crystal symmetry, so there is no contribution to the RD spectrum from the bulk.

The RDS technique was extended to probe surface states

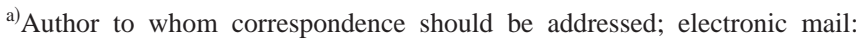
sjlee@ameslab.gov
}

of metallic cubic crystals such as (110) surfaces of $\mathrm{Cu}, \mathrm{Ag}$, and $\mathrm{Au}^{12-14}$ There have been no RD studies for metallic crystals having a noncubic structure. We have measured RD spectra for $\mathrm{R}_{5}\left(\mathrm{Si}_{x} \mathrm{Ge}_{1-x}\right)_{4}$ where $\mathrm{R}=\mathrm{Gd}$ and $\mathrm{Tb}$. The experimental data could contain both optical transitions between surface states, and between bulk states. The measured experimental data would provide useful information for developing theoretical models identifying the origin of optical transition mechanisms responsible for the observed reflectance anisotropy.

RD spectrum measurements were performed using a $75 \mathrm{~W}$ Xe short-arc lamp light source for a spectrum range from 1.5 to $5.5 \mathrm{eV}$. After passing a polarizer whose transmission axis is oriented along the vertical axis, the light becomes linearly polarized. This linearly polarized light passes through the photoelastic modulator (PEM) and experiences a periodically varying relative phase shift $\delta$ between orthogonal amplitude components. The relative phase shift has the form $\delta=\delta_{0} \sin \omega t$, where $\delta_{0}$ is the peak relative phase difference and $\omega$ is the modulation angular frequency of the PEM, which is $50 \mathrm{kHz}$ in our experiment. The reflected light from the surface of a sample passes through an analyzer whose transmission axis makes an angle of $45^{\circ}$ with respect to the vertical axis and goes into a $1 / 4 \mathrm{~m}$ monochromator. Finally, the light beam is detected by a photomultiplier. Lock-in amplifiers detect the modulated frequencies of 50 $\mathrm{kHz}(1 \omega)$ and $100 \mathrm{kHz}(2 \omega)$ from the photomultiplier.

The crystal structure of the $\mathrm{Gd}_{5} \mathrm{Si}_{2} \mathrm{Ge}_{2}$ compound contains 36 atoms per unit cell. ${ }^{15}$ The structure consists of twodimensional equivalent infinite Gd-containing slabs connected by $(\mathrm{Si}, \mathrm{Ge})$ dimers. At room temperature, $\mathrm{Gd}_{5} \mathrm{Si}_{2} \mathrm{Ge}_{2}$ crystallizes in the monoclinic structure ( $\beta$ phase, space group $P 112_{1} / a$ ) and at $276 \mathrm{~K}$, it transforms into an orthorhombic structure ( $\alpha$ phase, space group Pnma). The Gd-containing 
slabs are $a-c$ planes with the $b$ axis perpendicular to them for orthorhombic structure or almost perpendicular for monoclinic structure.

Single crystals of $a-b$ plane and $b-c$ plane of $\mathrm{Gd}_{5} \mathrm{Si}_{2} \mathrm{Ge}_{2}$ and $b-c$ plane of $\mathrm{Tb}_{5} \mathrm{Si}_{2.2} \mathrm{Ge}_{1.8}$ with dimensions $5 \times 4 \times 1 \mathrm{~mm}^{3}$ were grown by the Bridgeman method. Stoichiometric mixes of high purity gadolinium (99.996 wt \%), silicon (99.9999 wt \%), and germanium (99.999 wt \%) were cleaned and arc melted several times under an argon atmosphere. The as-cast ingot was electron-beam welded into a tungsten Bridgman style crucible for crystal growth. The ingot was then heated to $2000{ }^{\circ} \mathrm{C}$ and held at this temperature in molten state for $1 \mathrm{~h}$ to allow thorough mixing before withdrawing the sample from the heat zone at a cooling rate of 8 $\mathrm{mm} / \mathrm{h}$.

The definition of the reflectance difference spectrum is as follows:

$$
\mathrm{RD}(E)=\frac{\Delta R}{R}=\frac{R_{x}-R_{y}}{\left(R_{x}+R_{y}\right) / 2},
$$

where $R_{x}$ and $R_{y}$ are the reflectance intensity components along the $x$ and $y$ axis, respectively. The intensity measured at the detector is the square of the reflected electric field which can be calculated from the multiplication of Jones matrices and vectors representing the sample and optical components and incident polarized light. The reflected intensity can be decomposed into three components, $I_{\mathrm{dc}}, I_{\omega}$, and $I_{2 \omega}$. To a first-order approximation, the ratio of $I_{2 \omega}$ to $I_{\mathrm{dc}}$, which is related to reflectance difference, becomes

$$
\frac{I_{2 \omega}}{I_{\mathrm{dc}}}=\frac{2 J_{2}\left(\delta_{0}\right)\left(\left|\widetilde{r}_{x}\right|^{2}-\left|\widetilde{r}_{y}\right|^{2}\right) \sin 2 \theta}{\left|\widetilde{r}_{x}\right|^{2}+\left|\widetilde{r}_{y}\right|^{2}},
$$

where $J_{2}$ is a Bessel function of order 2 and $\theta$ is the angle between the orientation of the polarizer and the $y$ direction of the sample ( $b$ axis of the sample, the longest axis of the crystal unit cell $\left.{ }^{1}\right)$. We choose the amplitude of the modulation $\delta_{0}$ to satisfy $J_{0}\left(\delta_{0}\right)=0$, where $\delta_{0}=2.405 \mathrm{rad}$ or $137.8^{\circ}$.

From $R=\left(\left|\widetilde{r}_{x}\right|^{2}+\left|\widetilde{r}_{y}\right|^{2}\right) / 2$ and $\Delta R=\left(\left|\widetilde{r}_{x}\right|^{2}-\left|\widetilde{r}_{y}\right|^{2}\right)$, Eq. (2) becomes

$$
\frac{I_{2 \omega}}{I_{\mathrm{dc}}}=\frac{J_{2}\left(\delta_{0}\right) \Delta R \sin 2 \theta}{R} .
$$

The intensity ratio between the $2 \omega$ component and the dc component depends on the sample rotation angle $\theta$. One can define the angle-dependent reflectance difference $\operatorname{RD}(\theta)$ as

$$
\mathrm{RD}(\theta)=\frac{\Delta R}{R} \sin 2 \theta=\frac{1}{J_{2}\left(\delta_{0}\right)} \frac{I_{2 \omega}}{I_{\mathrm{dc}}} .
$$

The reflectance difference spectrum defined in Eq. (1) can be achieved when $\sin 2 \theta=1$. The incident polarized light beam was at an angle of incidence of less than $4^{\circ}$ to the sample surface normal. When $\theta$ is zero, the axis of the polarizer and $b$ axis of the samples are aligned. We rotated the sample about the $a$ axis of the $b-c$ plane sample and about the $c$ axis of the $a-b$ plane sample, with steps of $15^{\circ}$ or $30^{\circ}$. When the sample rotated $90^{\circ}$, the axis of the polarizer and the $b$ axis of the samples are perpendicular to each other.

Figures 1 and 2 show the angle-dependent reflectance difference spectra for the $b-c$ plane of $\mathrm{Gd}_{5} \mathrm{Si}_{2} \mathrm{Ge}_{2}$ and $a-b$ plane of $\mathrm{Gd}_{5} \mathrm{Si}_{2} \mathrm{Ge}_{2}$, respectively. For these measurements, the samples were rotated with a step of $30^{\circ}$ along an axis

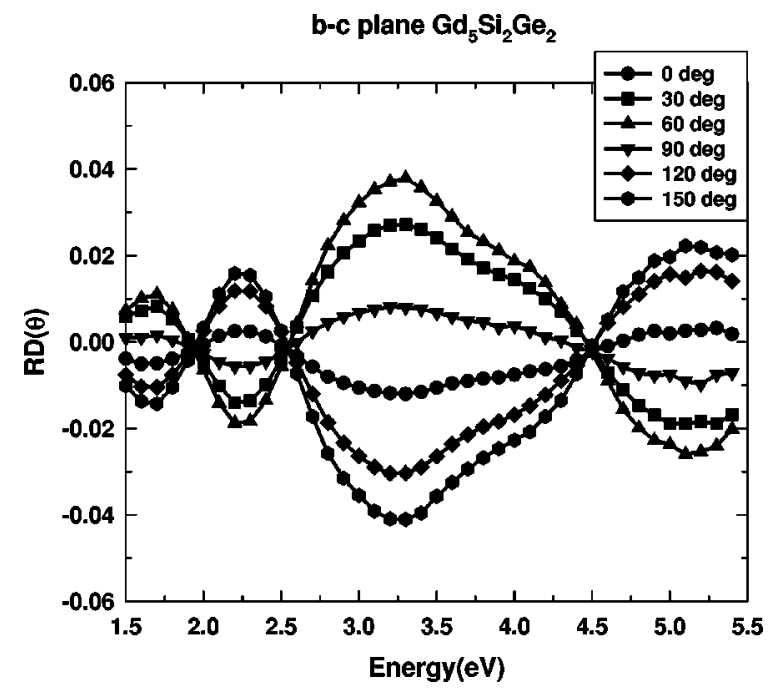

FIG. 1. Angle-dependent reflectance difference for the $b-c$ plane of $\mathrm{Gd}_{5} \mathrm{Si}_{2} \mathrm{Ge}_{2}$ with rotation angle from 0 to $150^{\circ}$ each $30^{\circ}$.

normal to the $b-c$ plane and $a-b$ plane $\mathrm{Gd}_{5} \mathrm{Si}_{2} \mathrm{Ge}_{2}$ sample, respectively. As shown in Figs. 1 and 2, noticeable peaks and zero crossings were observed in the spectra of the $b-c$ plane and $a-b$ plane of $\mathrm{Gd}_{5} \mathrm{Si}_{2} \mathrm{Ge}_{2}$, respectively. Although they have the same number of peaks, there are several noticeable differences in $\operatorname{RD}(\theta)$ between $b-c$ and $a-b$ planes of $\mathrm{Gd}_{5} \mathrm{Si}_{2} \mathrm{Ge}_{2}$. First, the small peak around $2.2 \mathrm{eV}$ in the $b-c$ plane sample becomes noticeably larger in the $a-b$ plane sample, and second, the peak around $3.2 \mathrm{eV}$ for the $b-c$ plane sample shifted to $3.7 \mathrm{eV}$ in the $a-b$ plane sample.

The angle-dependent reflectance difference spectrum of $b-c$ plane $\mathrm{Tb}_{5} \mathrm{Si}_{2.2} \mathrm{Ge}_{1.8}$, not shown, has similar features to that of $b-c$ plane $\mathrm{Gd}_{5} \mathrm{Si}_{2} \mathrm{Ge}_{2}$. Figure 3 shows the angledependent reflectance difference, $\operatorname{RD}(\theta)$, versus angle of sample rotation at fixed photon energy at which the largest amplitude of $\operatorname{RD}(\theta)$ occurs. As expected, the periodicities of $\operatorname{RD}(\theta)$ for the samples are $180^{\circ}$. The periodic sinusoidal behavior was not observed for the polycrystalline $\mathrm{Gd}_{5} \mathrm{Si}_{2} \mathrm{Ge}_{2}$ prepared by the arc-melted method using the same purity components due to the expected lack of anisotropy in a polycrystal compared with a single crystal.

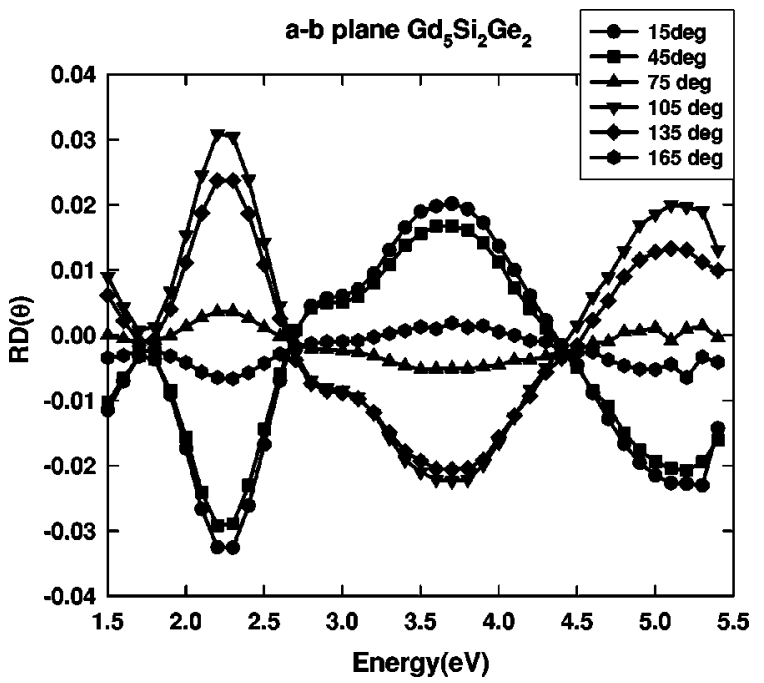

FIG. 2. Angle-dependent reflectance difference for the $a-b$ plane of $\mathrm{Gd}_{5} \mathrm{Si}_{2} \mathrm{Ge}_{2}$ with rotation angle from $15^{\circ}$ to $165^{\circ}$ each $30^{\circ}$. 


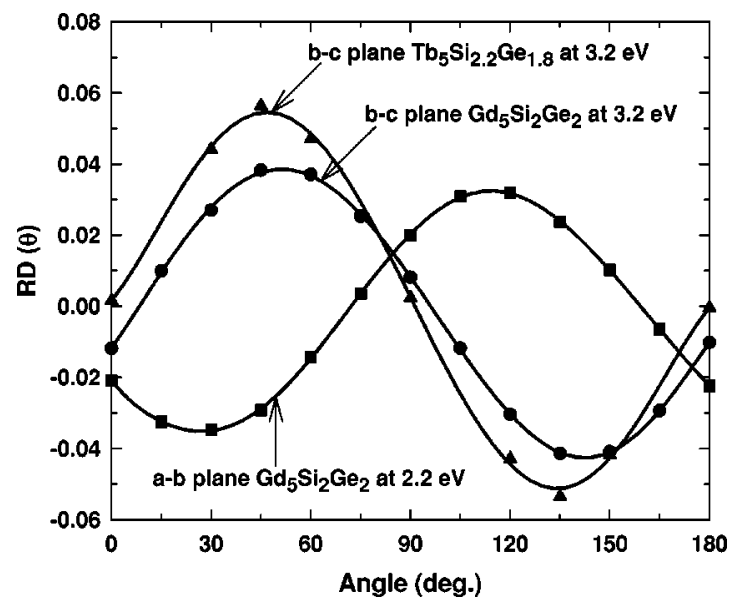

FIG. 3. Angle-dependent reflectance anisotropy for the $b-c$ plane of $\mathrm{Gd}_{5} \mathrm{Si}_{2} \mathrm{Ge}_{2}$ (closed circle) and $\mathrm{Tb}_{5} \mathrm{Si}_{2.2} \mathrm{Ge}_{1.8}$ (closed triangle), and the $a-b$ plane of $\mathrm{Gd}_{5} \mathrm{Si}_{2} \mathrm{Ge}_{2}$ (closed square) measured at fixed photon energies.

Peaks appeared at around $45^{\circ}$ and $135^{\circ}$ for $b-c$ plane $\mathrm{Gd}_{5} \mathrm{Si}_{2} \mathrm{Ge}_{2}$ and $\mathrm{Tb}_{5} \mathrm{Si}_{2.2} \mathrm{Ge}_{1.8}$ as shown in Fig. 3, which is consistent with the fact that $\sin 2 \theta$ becomes maximum at these angles. For the $a-b$ plane of $\mathrm{Gd}_{5} \mathrm{Si}_{2} \mathrm{Ge}_{2}$, the peak positions deviate slightly from the predicted values because the polarization axis and the $b$ axis of the samples were misaligned initially. The reflectance difference spectrum defined in Eq. (1) can be obtained by measuring the spectrum with a sample rotation angle at which the magnitude of $\operatorname{RD}(\theta)$ becomes maximum. We set the sample at this angle and measured the RD spectrum from 1.5 to $5.4 \mathrm{eV}$. The results are shown in Fig. 4. For both the $b-c$ plane of $\mathrm{Gd}_{5} \mathrm{Si}_{2} \mathrm{Ge}_{2}$ and $b-c$ plane of $\mathrm{Tb}_{5} \mathrm{Si}_{2.2} \mathrm{Ge}_{1.8}$, one big peak appeared at around $3.2 \mathrm{eV}$, a small peak at $1.7 \mathrm{eV}$, and a weak shoulder at $4.1 \mathrm{eV}$. For the $a-b$ plane of $\mathrm{Gd}_{5} \mathrm{Si}_{2} \mathrm{Ge}_{2}$, a weak shoulder at around $2.8 \mathrm{eV}$ and peaks at 1.7 and $3.8 \mathrm{eV}$ were observed. The similar features of the RD spectra for the $b-c$ plane of $\mathrm{Gd}_{5} \mathrm{Si}_{2} \mathrm{Ge}_{2}$ and $b-c$ plane of $\mathrm{Tb}_{5} \mathrm{Si}_{2.2} \mathrm{Ge}_{1.8}$ indicate that the RDS provides useful information about the optical anisotropy of samples, but is not an efficient probe for investigating the $4 f$ states of rare earths. This is due to the small dipole matrix elements for photoexciting $4 f$ electrons below $5 \mathrm{eV}$.

Because the penetration depth of the incident light is a

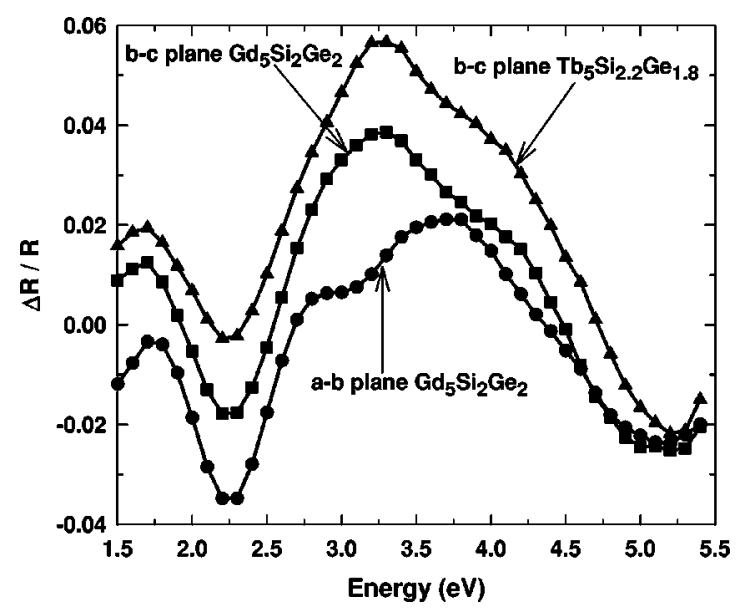

FIG. 4. Reflectance difference spectra for the $b-c$ plane of $\mathrm{Gd}_{5} \mathrm{Si}_{2} \mathrm{Ge}_{2}$ (closed square), $b-c$ plane of $\mathrm{Tb}_{5} \mathrm{Si}_{2.2} \mathrm{Ge}_{1.8}$ (closed triangle), and $a-b$ plane of $\mathrm{Gd}_{5} \mathrm{Si}_{2} \mathrm{Ge}_{2}$ (closed circle). few hundred angstroms for visible and UV light, the incident light can penetrate deep enough to interact with the atoms in the bulk. Therefore in analyzing RD spectra, optical transitions in terms of not only surface states but also bulk electronic states should be considered. Theoretical RD spectra from the electronic structures of surfaces and bulk would be useful in identifying the peaks in the spectrum and relative magnitude of surface and bulk anisotropy contributions to the signal.

In summary, we have measured $\mathrm{RD}$ spectra for single crystals of the $a-b$ plane and $b-c$ plane of $\mathrm{Gd}_{5} \mathrm{Si}_{2} \mathrm{Ge}_{2}$ and for a single crystal of the $b-c$ plane of $\mathrm{Tb}_{5} \mathrm{Si}_{2.2} \mathrm{Ge}_{1.8}$. The polarization axis of the incident light is parallel to the $b$ axis of the samples and the incident wave vector was near normal to the surface of the samples. We obtained an angledependent RD spectrum by rotating the sample in steps of $30^{\circ}$. At fixed photon energy, the angle-dependent RD had a sinusoidal form. From this sinusoidal curve, the angle at which $\operatorname{RD}(\theta)$ is a maximum can be obtained. The reflectance difference (RD) spectrum defined in Eq. (1) can be obtained by measuring a spectrum at this sample rotation angle.

The monoclinic crystal structure of the sample and the large penetration depth of the incident light leads to the conclusion that the contribution of bulk electronic optical transitions should be considered in addition to the surface electronic optical transitions to the RD signal. Probably all intermetallic surface states are destroyed due to the oxide layer on the free surface. They usually disappear from just one monolayer of an oxide. To fully analyze the experimental data, it is essential to develop first-principles calculations of the surface and bulk electronic structures from which theoretical RD spectrum would be obtained.

This manuscript has been authored by Iowa State University of Science and Technology under Contract No. W-7405-ENG-82 with the U.S. Department of Energy. The authors wish to thank Professor V.K. Pecharsky and Professor K.A. Gschneidner, Jr. for helpful discussion.

${ }^{1}$ V. K. Pecharsky and K. A. Gschneidner Jr., Phys. Rev. Lett. 78, 4494 (1997).

${ }^{2}$ V. K. Pecharsky and K. A. Gschneidner Jr., Appl. Phys. Lett. 70, 3299 (1997).

${ }^{3}$ L. Morellon, J. Stankiewicz, B. Garcia-Landa, P. A. Algarabel, and M. R. Ibrarra, Appl. Phys. Lett. 73, 3462 (1998).

${ }^{4}$ L. Morellon, P. A. Algarabel, M. R. Ibarra, J. Blasco, B. Garcia-Landa, Z. Arnold, and F. Albertini, Phys. Rev. B 58, R14721 (1998).

${ }^{5}$ V. Pecharsky and K. A. Gschneidner Jr., Adv. Mater. (Weinheim, Ger.) 13, 683 (2001).

${ }^{6}$ B. N. Harmon and V. N. Antonov, J. Appl. Phys. 91, 9815 (2002).

${ }^{7}$ G. D. Samolyuk and V. P. Antropov, J. Appl. Phys. 91, 8540 (2002).

${ }^{8}$ V. K. Pecharsky, G. D. Samolyuk, V. P. Antropov, A. O. Pecharsky, and K. A. Gschneidner, Jr., J. Solid State Chem. 171, 57 (2003).

${ }^{9}$ P. Weightman, D. S. Martin, and A. Maunder, J. Electron Spectrosc. Relat. Phenom. 114-116, 1069 (2001).

${ }^{10}$ Epioptics: Linear and Nonlinear Optical Spectroscopy of Surfaces and Interfaces, edited by J. F. McGilp and D. Weaire (Springer, Berlin, 1995).

${ }^{11}$ W. Richter and J.-T. Zettler, Appl. Surf. Sci. 100/101, 465 (1996).

${ }^{12} \mathrm{Ph}$. Hofmann, K. C. Rose, V. Fernandez, A. M. Bradshaw, and W. Richter, Phys. Rev. Lett. 75, 2039 (1995).

${ }^{13}$ Y. Borensztein, W. I. Mochan, J. Tarriba, R. G. Barrera, and A. Tadjeddine, Phys. Rev. Lett. 71, 2334 (1993).

${ }^{14}$ K. Stahrenberg, Th. Herrmann, N. Esser, W. Richter, S. V. Hoffmann, and Ph. Hofmann, Phys. Rev. B 65, 035407 (2001).

${ }^{15}$ W. Choe, V. K. Pecharsky, A. O. Pecharsky, K. A. Gschneidner Jr., V. G. Young, and G. J. Miller, Phys. Rev. Lett. 84, 4617 (2000). 